\title{
ASPECTOS TAXONÓMICOS Y DE CONSERVACIÓN DE LA TRIBU TRICHOCEREEAE EN EL CERRO UMARCATA Y QUEBRADA OROBEL, VALLE DEL RÍO CHILLON, LIMA
}

\section{TAXONOMIC AND ECOLOGICAL ASPECTS OF TRICHOCEREEAE TRIBE AT UMARCATA HILL AND OROBEL VALLEY, CHILLÓN RIVER VALLEY, LIMA}

\author{
Viviana Castro-Cepero ${ }^{1}$ y Aldo Ceroni-Stuva ${ }^{2}$
}

\begin{abstract}
Resumen
Se presenta el estudio de la taxonomía y estado de conservación de tres cactáceas dentro de la tribu Trichocereeae de la familia Cactaceae: Cleistocactus acanthurus subsp. faustianus (Backeberg) Ostolaza, Espostoa melanostele (Vaupel) Borg y Mila nealeana Backeberg, ubicadas en el cerro Umarcata y la quebrada Orobel, en la cuenca baja del río Chillón. Las plantas corresponden a colectas realizadas entre los años 2003 y 2004 en el marco de un proyecto de investigación sobre las cactáceas de Lima. Se registraron datos sobre las características taxonómicas de las plantas, así como su ubicación y su estado de conservación. Cleistocactus acanthurus subsp. faustianus, cactus decumbente que alcanza los 40 a $45 \mathrm{~cm}$ de altura, se distribuye en los valles de Chillón, Rímac y Santa Eulalia (Lima), se encuentra en estado de En Peligro (EN). Espostoa melanostele, cactus columnar que alcanza entre 1.3 y $2 \mathrm{~m}$ de altura, se localiza desde el valle de Saña (Lambayeque) hasta el valle de Pisco (Ica), se clasifica en la categoría de Casi Amenazado (NT). Mila nealeana, cactus cespitoso que alcanza entre 8 a $10 \mathrm{~cm}$ de altura, endémico de Lima, encontrado desde los valles de Fortaleza hasta Cañete, y catalogado como En Peligro (EN), según lo establecido en el Decreto Supremo Nº46-2006-AG.
\end{abstract}

Palabras clave: taxonomía, cactus, cactaceae, conservación, Chillón.

\begin{abstract}
The study of taxonomy and conservation status of three cacti inside of the Trichocereeae tribe of the Cactaceae family is presented: Cleistocactus acanthurus subsp. faustianus (Backeberg) Ostolaza, Espostoa melanostele (Vaupel) Borg and Mila nealeana Backeberg, located in Umarcata hill and Orobel Valley, in the lower basin of the Chillón River. Plants correspond to collections made between 2003 and 2004 as part of a larger research project on the Cactaceae of Lima. Data on the taxonomic characteristics of plants and their location and condition were recorded. Cleistocactus acanthurus subsp. faustianus, decumbent cactus that reaches $40-45 \mathrm{~cm}$ in height, is located in Chillón, Rímac and Santa Eulalia valleys (Lima) and is in Endangered (EN) status. Espostoa melanostele, columnar cactus reaching between 1.3 and $2 \mathrm{~m}$ high, is located from Saña (Lambayeque) to Pisco (Ica) valleys and is in Near Threatened (NT) status, while Mila nealeana, cespitose cactus reaching 8-10 cm tall, endemic to Lima, is located from Cañete to Fortaleza valleys and is in Endangered (EN) status, according to D.S. $\mathrm{N}^{\circ} 046-2006-A G$.
\end{abstract}

Key words: Taxonomy, cacti, cactaceae, conservation, Chillon.

\section{Introducción.}

La familia Cactaceae en el Perú está representada por 34 géneros y más de 255 especies (Hunt, 1999). Estas plantas están distribuidas en casi todos los ecosistemas, desde los desiertos costeros, vertiente occidental, puna, valles interandinos y llegando hasta el bosque tropical amazónico (Calderón et al., 2004). Las cactáceas conforman los elementos característicos en la formación vegetal conocida como Piso de Cactáceas Columnares, formación xerofítica ubicada al centro y sur de la vertiente occidental del Perú, dominada por arbustos y cactáceas columnares
(Weberbauer, 1945). Dentro de los ecosistemas áridos de Lima, el cerro Umarcata y la quebrada Orobel son zonas de alta diversidad específica de cactáceas (Teixeira et al., 2004). En efecto, estudios realizados en dichas zonas sobre la taxonomía, diversidad, densidad, fenología e interacciones de estas plantas, han resaltado su rol como especies clave en ecosistemas áridos (Castro, 2006; Teixeira et al., 2004; Novoa et al., 2005a; 2005b y 2005c); esto pone en evidencia además que las zonas áridas de la costa central del Perú, son áreas importantes a conservar por ser lugares de alta diversidad de estas especies y 
Enero - Julio 2015

también por los futuros trabajos de investigación que puedan hacerse a fin de entender mejor su dinámica y favorecer su gestión (Ceroni \& Castro, 2006). Por último la pérdida de estos espacios, que son reemplazados por zonas urbanas y agrícolas, ha desencadenado la implementación de iniciativas puntuales para lograr su conservación ex situ (Ceroni et al., 2004).

Las cactáceas que se encuentran en estos lugares son nueve especies comprendidas en 8 géneros y 2 subfamilias (Castro, 2006; Calderón et al., 2007). En la subfamilia Cactoidea: Melocactus peruvianus Vaupel, Cleistocactus acanthurus subsp. faustianus (Backeberg) Ostolaza, Espostoa melanostele (Vaupel) Borg, Haageocereus acranthus (Vaupel) Backeberg, Haageocereus pseudomelanostele subsp. aureispinus (Rauh \& Backeberg) Ostolaza, Mila nealeana Backeberg y Neoraimondia arequipensis subsp. roseiflora (Werdermann \& Backeberg) Ostolaza; mientras que en la subfamilia Opuntioideae: Austrocylindropuntia pachypus (Schumann) Backeberg y Cumulopuntia sphaerica (Förster) Anderson. En la presente nota científica, se presentan algunos aspectos taxonómicos y estado de conservación de tres especies de la subfamilia Cactoideae, dentro de la Tribu Trichocereeae: Mila nealeana, Espostoa melanostele y Cleistocactus acanthurus subsp. faustianus.

\section{Materiales y métodos.}

Área de estudio.

La primera zona de colecta, llamada Cerro Umarcata, se encuentra a una distancia de 6 kilómetros del centro del distrito de Santa Rosa de Quives, a la altura del kilómetro 69 de la carretera Lima - Canta, a una altitud de 1260 msnm y a 11³7'39', LS y 7646'9', LO. La segunda zona de colecta, llamada Quebrada Orobel, se encuentra a una distancia de 4 kilómetros de Quives, a la altura del kilómetro 4 de la carretera a Arahuay, a una altitud de 1380 msnm y a 11³9'37', LS y 7645'26', LO. Ambas zonas se encuentran dentro de la formación vegetal conocida como Piso de Cactáceas Columnares (1 100 a $2400 \mathrm{msnm}$ ) del centro de la vertiente occidental del Perú (Figura 1). El análisis de suelo realizado en el Laboratorio de Análisis de Suelos y Plantas de la Universidad Nacional Agraria La Molina, dio un pH que varía entre 5 y 7.6; la conductividad eléctrica varía entre 0.69 y $1.96 \mathrm{dS} / \mathrm{m}$; el porcentaje de carbonato de calcio alcanza un valor máximo de 1.3; el porcentaje de materia orgánica varía entre 1.3 y 3.6; el contenido de fósforo oscila entre 22.2 y 41.3 ppm y el contenido de potasio, entre 340 y 860 ppm.

Colección botánica.

Se colectaron las especies de cactáceas presentes en el Cerro Umarcata y Quebrada Orobel, distrito de Santa Rosa de Quives, en el Valle del Río Chillón,

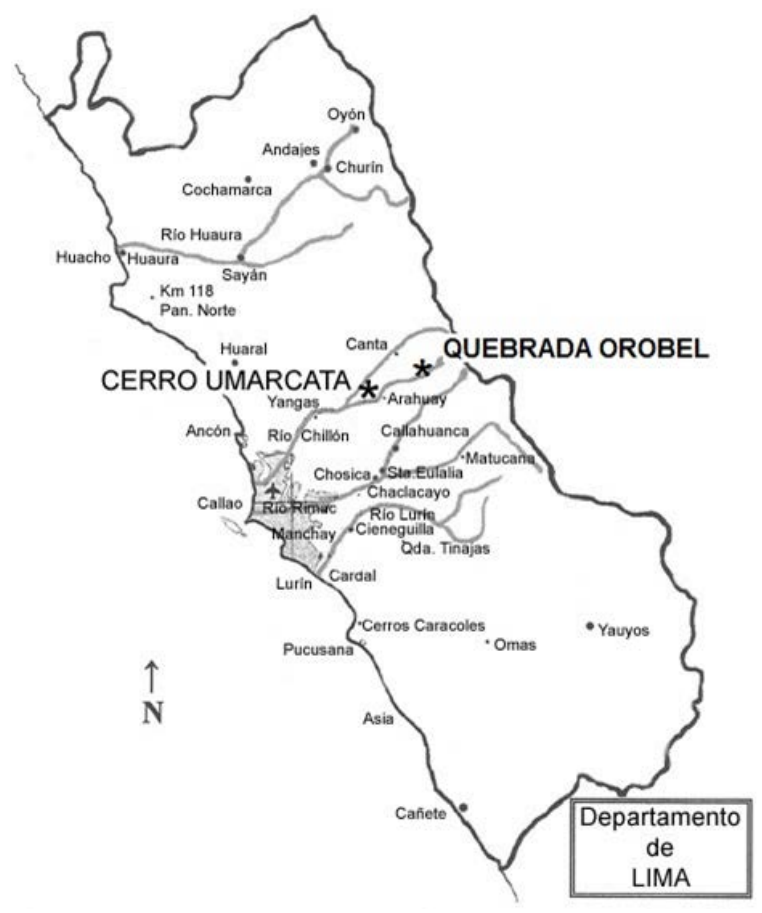

Figura 1. Mapa de la zona de estudio: Cerro Umarcata y Quebrada Orobel.

registrándose los datos de cada individuo en las etiquetas y libretas de campo, así como datos de ubicación, entre otros. Las plantas fueron recolectadas al azar en los meses de febrero y julio de 2003 y febrero, mayo, agosto y diciembre de 2004. Se colectó casi toda la planta haciendo un corte en la parte basal. Las flores y frutos se depositaron en frascos o sobres, identificadas apropiadamente y tratadas en el gabinete. Las plantas fueron depositadas en el Herbario de Sitio del Jardín Botánico “Octavio Velarde Núñez” de la Universidad Nacional Agraria La Molina, debido a que este Jardín Botánico cuenta con una colección de cactáceas de Lima.

Estudio taxonómico.

Las muestras colectadas fueron tratadas en el Centro de Interpretación "Casa Julio Gaudron" del Jardín Botánico "Octavio Velarde Núñez" de la Universidad Nacional Agraria La Molina, siguiendo los métodos sugeridos por Baker et al. (1985). Se cortó el espécimen de la base al ápice, aislando las costillas, en secciones longitudinales y transversales. Todas las muestras tomadas se llevaron al secador en una prensa convencional, cambiando el papel con frecuencia y utilizando para la preservación la solución FAA y bórax (fungicida). Los frutos se prensaron aparte $\mathrm{y}$ fueron cortados en secciones longitudinales y transversales. Antes de seccionar el material, se registraron las dimensiones de cada individuo. El material fértil sirvió para la determinación taxonómica del material recolectado (flores, frutos y semillas) y fue preservado en frascos 
con alcohol o congelado, hasta su uso. La determinación taxonómica se realizó en base a claves de determinación y en base a la comparación con muestras conservadas en los herbarios de la Universidad Nacional Agraria La Molina (MOL) y Universidad Nacional Mayor de San Marcos (UNMSM).

\section{Resultados.}

Subfamilia Cactoideae Buxbaum.

Plantas arborescentes, arbustivas, cespitosas, trepadoras o epífitas; raíces fibrosas o pivotantes; tallos ramificados desde la base, parte media o ápice, no segmentados, globosos o columnares, con costillas y tubérculos; zonas reproductivas no diferenciadas o diferenciadas en cefalios; hojas vestigiales o ausentes; gloquidios ausentes; flores sésiles, diurnas o nocturnas; pericarpelo desnudo o con escamas y areolas; tubo floral corto o elongado; areolas florales con pelos, espinas o cerdas; estambres numerosos insertos en el tubo del perianto; frutos secos o carnosos, de tamaño y forma variable; semillas ovaladas o circulares a veces con expansiones y testa de arquitectura variada; arilo ausente (Anderson, 2001; Barthlott \& Hunt, 1993; Hunt et al., 2006). La subfamilia comprende las tribus Trichocereeae, Brownngieae y Cereeae, las cuales están estrechamente relacionadas a nivel genético (Wallace \& Gibson, 2002) y conforman el clado "BCT". De acuerdo a Taylor \& Zappi (2004), considerando delimitaciones a nivel morfológico, Trichocereeae está aparentemente más relacionado a Cereeae que a Browningieae, debido a que Cereeae presenta flores desnudas y Trichocereeae presenta flores cubiertas de pelos, espinas y cerdas.

Tribu Trichocereeae Buxbaum.

Plantas de hábito arborescente o arbustivo; tallos normalmente no segmentados, globosos o columnares, con costillas, costillas tuberculadas o tubérculos; zonas reproductivas diferenciadas en cefalios laterales o apicales o no diferenciadas; flores sésiles, laterales o subapicales, diurnas o nocturnas, actinomorfas o zigomorfas; pericarpelo con escamas o pelos; frutos carnosos; semillas ovaladas sin expansiones (Anderson, 2001; Barthlott \& Hunt, 1993).

Mila Britton \& Rose. The Cactaceae; descriptions and illustrations of plants of the cactus family 3: 211 . 1922.

Planta arbustiva baja, cespitosa. Tallo terete, formando grupos, cilíndrico, con 10 a 13 costillas, areolas pequeñas, con espinas variables. Flor subapical, campanulada a infundibuliforme, de tubo corto y estrecho, amarilla, estambres epipétalos, pericarpelo y tubo floral cubiertos de pelos y espinas finas. Fruto pequeño, globoso, jugoso, rojo tinto, con pelos y espinas, semillas numerosas y negras. Género endémico de Lima. En la zona de estudio se determinó una especie.

\section{Mila nealeana Backeberg. Blätter für} Kakteenforschung 1(1): 2. 1934.

Sinónimos: Mila alboareolata Akers. M. breviseta Rauh \& Backeberg. M. caespitosa fo. breviseta (Rauh \& Backeberg) Donald. M. caespitosa fo. kubeana (Werdermann \& Backeberg) Donald. M. caespitosa fo. lurinensis (Rauh \& Backeberg) Donald. $M$. caespitosa subsp. nealeana (Backeberg) Donald. $M$. caespitosa var. alboareolata (Akers) Donald. $M$. caespitosa var. densiseta (Rauh \& Backeberg) Donald. M. densiseta Rauh \& Backeberg. M. kubeana Werdermann \& Backeberg. M. lurinensis Rauh \& Backeberg. $M$. nealeana fo. senilis F. Ritter. $M$. nealeana var. tenuior Rauh \& Backeberg. $M$. sublanata var. pallidior Rauh \& Backeberg.

Planta cespitosa que alcanza entre 8 a $10 \mathrm{~cm}$ de altura, ramificada desde la base, formando almohadillas, con algunos tallos erectos y otros postrados. Raíz napiforme. Tallo terete de 2 a $3 \mathrm{~cm}$ de diámetro, con 12 a 15 costillas, epidermis visible y verde a verde grisáceo; areolas de forma redonda, de $0.1 \mathrm{~cm}$ de espesor y $0.2 \mathrm{~cm}$ de diámetro, color gris; espinas radiales aciculares, 15 a 20 espinas por areola, de $1.2 \mathrm{~cm}$ de longitud, $0.1 \mathrm{~cm}$ de grosor, de color gris; espina central acicular, entre 2.3 y $3 \mathrm{~cm}$ de longitud, $0.1 \mathrm{~cm}$ de grosor y 3 a 5 espinas por areola, grises; flores actinomorfas, infundibuliformes de tubo corto, amarillas, desarrollándose sobre las areolas floríferas, próximas al ápice del tallo, entre 2.2 y $3 \mathrm{~cm}$ de longitud y perianto de 0.9 a $2.2 \mathrm{~cm}$ de diámetro, con aproximadamente 30 a 40 tépalos: sepaloide, lanceolado-apiculado, de 0.6 a $1.15 \mathrm{~cm}$ de longitud y de 0.1 a $0.3 \mathrm{~cm}$ de ancho, y petaloide linguladocuspidado, de 1.25 a $1.3 \mathrm{~cm}$ longitud y $0.35 \mathrm{~cm}$ de ancho; tubo del receptáculo, de 0.7 a $1.3 \mathrm{~cm}$ de longitud y de 0.4 a $0.6 \mathrm{~cm}$ de diámetro, cubierto con brácteas a manera de escamas y pelos lanosos cortos; cámara nectarial de 0.2 a $0.3 \mathrm{~cm}$ de longitud y $0.2 \mathrm{~cm}$ de diámetro; androceo con 90 a 120 estambres epipétalos aproximadamente, entre 0.55 y $0.81 \mathrm{~cm}$ de longitud, anteras bitécicas de $0.1 \mathrm{~cm}$ de longitud y $0.05 \mathrm{~cm}$ de diámetro, amarillas, filamentos entre 0.45 y $0.7 \mathrm{~cm}$ de longitud y $0.025 \mathrm{~cm}$ de diámetro, color marfil; pericarpelo de 0.4 a $0.5 \mathrm{~cm}$ de longitud y de 0.35 a $0.5 \mathrm{~cm}$ de diámetro, cubierto con pequeñas brácteas a manera de escamas y pelos lanoso cortos; gineceo sincárpico de 1.55 a $2.2 \mathrm{~cm}$ de longitud, ovario ínfero de 0.25 a $0.4 \mathrm{~cm}$ de longitud y entre 0.25 a $0.35 \mathrm{~cm}$ de diámetro, 8 carpelos, numerosos óvulos en placentación parietal, estilo de 0.9 a $1.3 \mathrm{~cm}$ de longitud y de 0.05 a $0.1 \mathrm{~cm}$ de diámetro, estigma lobulado de 0.25 a $0.4 \mathrm{~cm}$ de longitud y $0.35 \mathrm{~cm}$ de diámetro, de 7 a 9 lóbulos color verde; fruto baya, de color verde a rojo oscuro o guinda, ovoide de 1.2 a $1.5 \mathrm{~cm}$ de longitud, de 0.8 a $0.9 \mathrm{~cm}$ de diámetro mayor y de 0.3 a $0.5 \mathrm{~cm}$ de diámetro menor, con flor persistente de $1.5 \mathrm{~cm}$ de longitud, funículo ariloso blanco transparente. Según Ramírez \& Ceroni (2009) 


\section{LA TRIBU TRICHOCEREEAE EN EL VALLE DEL RÍO CHILLÓN}

Enero - Julio 2015

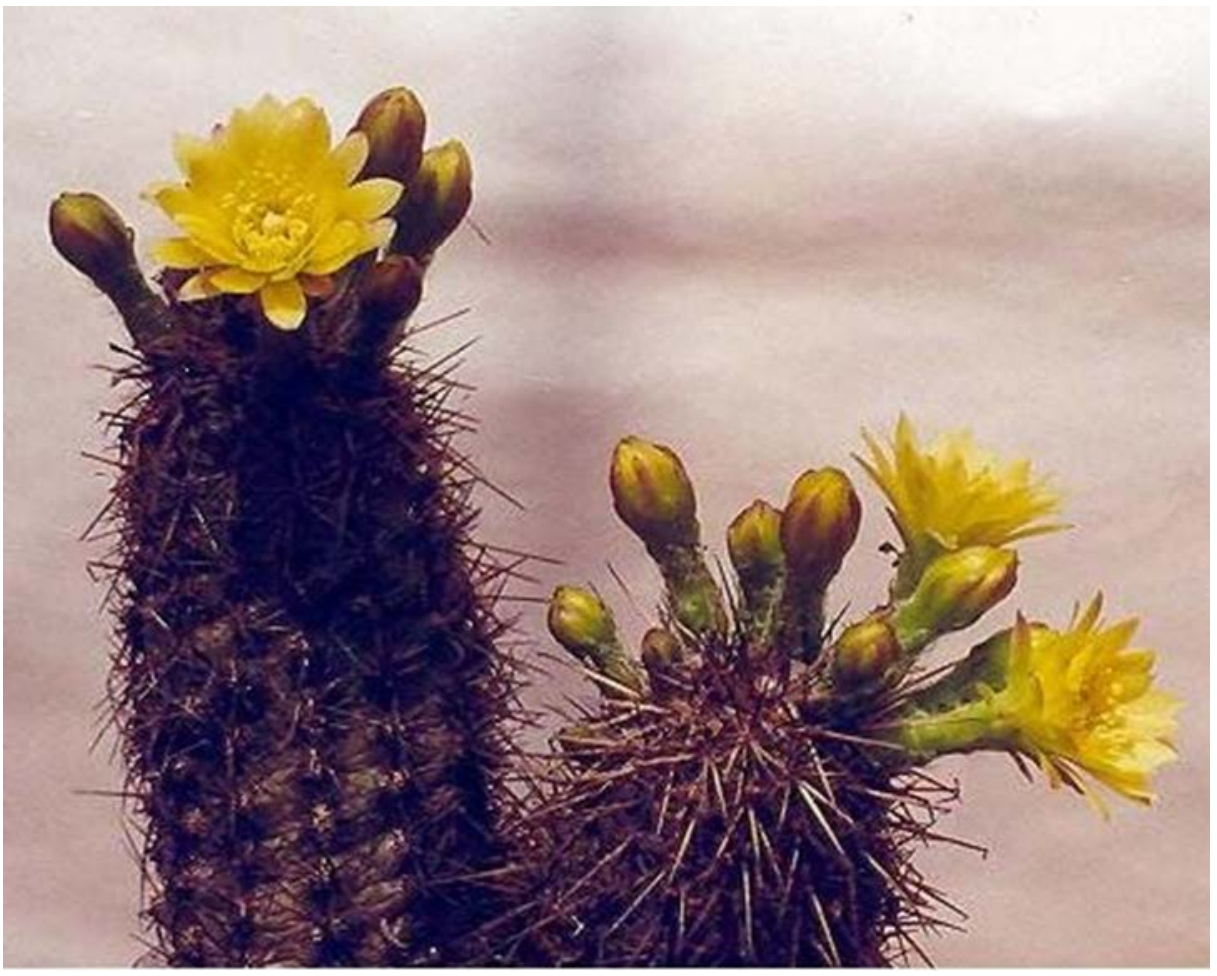

Figura 3. Mila nealeana Backeberg.

las semillas son de forma ovalado-reniforme, con testa de color negro-marrón, de tamaño mediano de 1.12 mm de longitud y $0.68 \mathrm{~mm}$ de ancho; la escultura de la testa es ruminada, con fuertes estriaciones cuticulares en el microrelieve; la región hilomicropilar es grande y oblicua con el eje mayor de la semilla, con micrópilo e hilo grandes; los frutos presentan entre 23 a 248 semillas (Figura 2). Especie endémica de Lima, abarcando desde los valles de Fortaleza hasta Cañete.

Material estudiado: LIMA: Cerro Umarcata, Santa Rosa de Quives, julio de 2003, 1260 msnm, Viviana Castro 111 (Herbario de Sitio Jardín Botánico OVN); Quebrada Orobel, Santa Rosa de Quives, diciembre de 2004, 1380 msnm, Viviana Castro 122 (Herbario de Sitio Jardín Botánico OVN); Canta, noviembre de 1979, 700 - 800 msnm, C. Ostolaza, 003 (USM) (Figura 3).

El género fue publicado por Britton \& Rose (19191922) como un anagrama de Lima, donde fue colectado; la especie fue publicada en 1922 bajo el nombre de Mila caespitosa Britton \& Rose y a pesar de encontrar otras plantas muy distintas a la descrita, las incluyeron en esta nueva especie al no poder encontrar material fértil. Backeberg en su afán de dividir los taxones, publica en 1934 la especie Mila nealeana, basándose en las leves diferencias vegetativas y reproductivas encontradas por Britton \& Rose (1919-1922) y que no pudieron ser contrastadas. Es registrada luego por Brako \& Zarucchi (1993) como Mila nealeana Backeberg. Posteriormente es tratada por Ostolaza (1996), Hunt (1999) y Ostolaza et al. (2003 y 2005) bajo el nombre de Mila nealeana Backeberg. Anderson (2001) y Hunt et al. (2006) la tratan como Mila caespitosa Britton \& Rose. Anderson (2001) enfatiza que este género debe ser reconocido como monoespecífico, con gran variabilidad entre sus individuos y basa su propuesta en estudios moleculares que muestran que no existe variación en el ADN entre las supuestas especies del género Mila. Más recientemente Ostolaza (2011) la considera como Mila nealeana Backeberg con distribución en el Valle del Rímac y valles laterales, lo cual es recogido por la base de datos TROPICOS (2014). Por último, se encuentra registrada en la base de datos ITIS como Mila caespitosa subsp. caespitosa (ITIS, 2014). A pesar de las discrepancias nomenclaturales existentes, hasta la fecha no existe publicación que respalde la propuesta de considerar Mila como género monoespecífico, por lo que se debe respetar el último taxón válidamente publicado y este corresponde al de Mila nealeana Backeberg.

Según Arakaki et al. (2006) esta especie es válida y presenta estado de conservación No Evaluado; de acuerdo a la normatividad legal vigente, se encuentra en estado de Amenazada (EN) (D.S.043, 2006). Así mismo, ha sido considerada por Ostolaza \& Cáceres (2013) en estado Vulnerable (V), debido al avance de las zonas urbanas y agrícolas en las cercanías de Lima, con evidencias de que las poblaciones se encuentran en disminución.

Espostoa Britton \& Rose. The Cactaceae; descriptions and illustrations of plants of the cactus family 2: 60, f. 87-91. 1920.

Planta arbustiva o arborescente, columnar. Tallo terete, con 4 a 30 costillas, areolas circulares con espinas y pelos largos. Cefalio lateral, formado por algunas costillas reducidas y modificadas, cubierto de pelos lanosos, blancos, cremosos o marrones y espinas. Flor tubular o acampanada, que nace en el cefalio, nocturna, rojiza a blanca, pequeña, tubo floral corto, pericarpelo y tubo floral con pequeñas brácteas 
a manera de escamas y pelos, nunca con espinas, ovario ínfero. Fruto globular a ovoide, jugoso, verde a rojo, con flor persistente, semillas marrones. Distribución: sur de Ecuador, Perú y Bolivia. En la zona de estudio se determinó una especie.

Espostoa melanostele (Vaupel) Borg. Cacti 112. 1937. Cephalocereus melanostele Vaupel. Botanische Jahrbücher für Systematik, Pflanzengeschichte und Pflanzengeographie 50(2-3, Beibl. 111): 12-13. 1913.

Sinónimos: Binghamia melanostele (Vaupel) Britton \& Rose. Cereus melanostele (Vaupel) Werdermann. Espostoa melanostele fo. inermis (Backeberg) Krainz. Haageocereus melanostele (Vaupel) W.T. Marshall. Pseudoespostoa melanostele (Vaupel) Backeberg. P. melanostele var. inermis Backeberg.

Planta columnar que alcanza entre 1.3 y 2 m de altura, ramificada desde la base, formando grupos de numerosos tallos. Tallo terete de 8 a $10 \mathrm{~cm}$ de diámetro, con 20 a 25 costillas, epidermis verde cubierta por pelos lanosos blancos a marrones de 1 a 2 $\mathrm{cm}$ de longitud; areolas cubiertas por los mismos pelos, de forma redonda, de 0.1 a 0.2 de espesor y de 0.2 a $0.5 \mathrm{~cm}$ de diámetro, color negro; espinas radiales aciculares, 40 a 50 espinas por areola, de 0.5 a $1 \mathrm{~cm}$ de longitud, 0.05 a $0.1 \mathrm{~cm}$ de grosor, de color amarillo al aparecer y negro al madurar; espina central acicular, entre 4 a $10 \mathrm{~cm}$ de longitud, $0.1 \mathrm{~cm}$ de grosor y 1 a 3 espinas por areola, amarillas; cefalio lateral, de pelos lanosos blancos, amarillos o marrones, de 50 a $70 \mathrm{~cm}$ de longitud, cubriendo aproximadamente 8 costillas; flores actinomorfas, acampanadas, blancas perlado, desarrollándose en el cefalio lateral, entre 3.9 y $5.2 \mathrm{~cm}$ de longitud y perianto de 1.5 y 2 de diámetro, con aproximadamente 45 tépalos: sepaloide, lanceoladoapiculado, de 0.5 a $1.5 \mathrm{~cm}$ de longitud y de 0.2 a 0.4 cm de ancho, y petaloide lingulado-rotundo, de 0.8 a $1.5 \mathrm{~cm}$ longitud y de 0.4 a $0.65 \mathrm{~cm}$ de ancho; tubo del receptáculo, de 2.5 a $3.7 \mathrm{~cm}$ de longitud y de 1 a 1.5 cm de diámetro, cubierto con pelos lanosos cortos y blancos; cámara nectarial de 0.6 a $1 \mathrm{~cm}$ de longitud y de 0.6 a 0.9 cm de diámetro; androceo con aproximadamente 300 estambres, entre 0.7 y $2 \mathrm{~cm}$ de longitud, anteras bitécicas entre 0.2 y $0.3 \mathrm{~cm}$ de longitud y $0.1 \mathrm{~cm}$ de diámetro, amarillas, filamentos entre 0.5 y $1.7 \mathrm{~cm}$ de longitud y $0.05 \mathrm{~cm}$ de diámetro, color marfil; pericarpelo de $1 \mathrm{~cm}$ de longitud y de 0.9 a $1.2 \mathrm{~cm}$ de diámetro, cubierto con pequeñas brácteas a manera de escamas; gineceo sincárpico de 3.4 a 5.2 $\mathrm{cm}$ de longitud, ovario ínfero de 0.4 a $0.5 \mathrm{~cm}$ de longitud y de 0.5 a $0.6 \mathrm{~cm}$ de diámetro, 9 carpelos, numerosos óvulos en placentación parietal, estilo de 2.2 a $3.7 \mathrm{~cm}$ de longitud y de 0.15 a $0.2 \mathrm{~cm}$ de diámetro, estigma lobulado de 0,3 a $0.5 \mathrm{~cm}$ de longitud y 0.4 a $0.8 \mathrm{~cm}$ de diámetro, 15 lóbulos color blanco verdoso; fruto baya, de color verde, blanco o rojo, esférico, de 3 a $5 \mathrm{~cm}$ de diámetro, funículo ariloso blanco transparente desarrollado. Según
Ramírez \& Ceroni (2009) las semillas son de forma ovalada, con testa de color negro lustroso, de tamaño mediano de $1.2 \mathrm{~mm}$ de longitud y $0.88 \mathrm{~mm}$ de ancho; la escultura de la testa es ligeramente ruminada; la región hilo-micropliar es grande y oblicua con el eje mayor de la semilla, con micrópilo e hilo grandes; los frutos presentan entre 600 a 900 semillas (Figura 4).

La especie se distribuye desde el valle de Saña (Lambayeque) hasta el valle de Pisco (Ica).

Material estudiado: LIMA: Cerro Umarcata, Santa Rosa de Quives, diciembre de 2004, 1260 msnm, Viviana Castro 121 (Herbario de Sitio Jardín Botánico OVN); Santa Eulalia, 1625 msnm, noviembre de 1964, P. Hutchinson 7049 (USM); Huaura, agosto de 2003, 1120 msnm, Mónica Arakaki, 1557 (USM); Oyón, agosto de 2003, 2880 msnm, Mónica Arakaki, 1564 (USM) (Figura 5).

De acuerdo a Britton \& Rose (1919-1922) la especie fue descrita como Cephalocereus melanostele Vaupel en 1913. En 1920 Britton \& Rose publican el género Binghamia, para establecer la diferencia con el género Cephalocereus el cual es originario de México; es así que la especie fue publicada en 1920 como Binghamia melanostele (Vaupel) Britton \& Rose. Sin embargo, en este mismo año Britton \& Rose publicaron también el género Espostoa, como el equivalente local del género mexicano Cephalocereus, el cual se caracteriza por los abundantes pelos lanosos que produce y las flores campanuladas blancas de tubo corto. Luego Borg (1959) utiliza este nuevo género para ubicar la especie Espostoa melanostele. Es por esto que posteriormente es tratada y registrada por Brako \& Zarucchi (1993), Hunt (1999), Anderson (2001), Ostolaza et al. (2003 y 2005), Hunt et al. (2006), Ostolaza (2011), TROPICOS (2014) e ITIS (2014) como Espostoa melanostele (Vaupel) Borg, la cual se caracteriza principalmente por la presencia de un cefalio lateral con pelos lanosos que contiene flores blancas nacaradas de tubo corto, con distribución en los valles costeros de Lambayeque, La Libertad, Ancash, Lima e Ica, desde los 800 a 1500 msnm.

Según Arakaki et al. (2006) esta especie es válida y presenta estado de conservación No Evaluado; de acuerdo a la normatividad legal vigente, se encuentra en estado de Casi Amenazado (NT) (D.S.043, 2006). Así mismo, ha sido considerada por Cáceres et al. (2013) en estado de Preocupación Menor (LC), debido al amplio rango de ocurrencia y a que no está expuesta a ninguna amenaza, lo que se refleja en una población estable.

Cleistocactus Lemaire. L'illustration horticole 8: Misc. 35. 1861.

Planta arbustiva, rara vez arborescente, decumbente. Tallo terete, postrado, con ápices ascendentes o péndulos, delgados, 5 a 30 costillas, casi sin tubérculos, a veces con surcos transversales. Areolas muy juntas, a veces las areolas florales con pelos lanosos y cerdas. Espinas variables. Flores que 


\section{LA TRIBU TRICHOCEREEAE EN EL VALLE DEL RÍO CHILLÓN}

Enero - Julio 2015

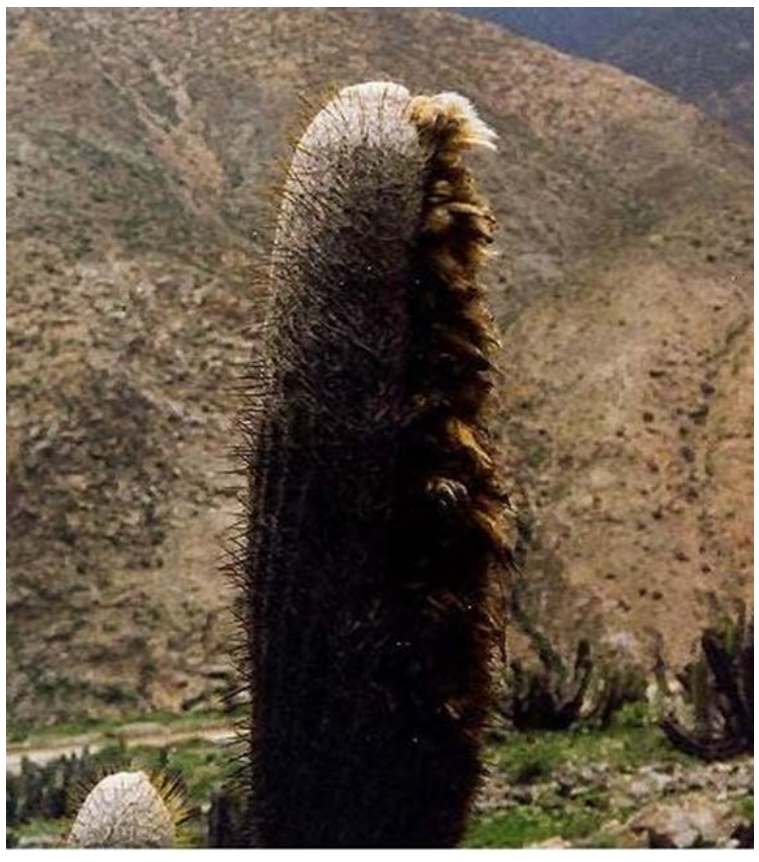

Figura 5. Espostoa melanostele (Vaupel) Borg.

aparecen lateralmente en la porción superior del tallo, abundantes, diurnas, rojas, anaranjadas, amarillas o verdes; flor tubular, de tubo estrecho, recto, curvo o en forma de S, perianto no expandido, estambres exertos o no, usualmente en dos ciclos, estilo exerto, pericarpelo y tubo floral cubierto por brácteas a manera de escamas, pelos lanosos y espinas, ovario ínfero. Fruto pequeño, globular, jugoso, con flor persistente, semillas negras. Distribución: sur de Ecuador, oeste de Brasil, norte de Argentina, Perú, Bolivia, Uruguay y Paraguay.

Cleistocactus acanthurus (Vaupel) Hunt. Bradleya; Yearbook of the British Cactus and Succulent Society 5. 1987. Cereus acanthurus Vaupel. Botanische Jahrbücher für Systematik, Pflanzengeschichte und Pflanzengeographie 50: 1314. 1913.

Sinónimo: Binghamia acanthura (Vaupel) Borg, B. eriotricha (Werdermann \& Backeberg) Borg, Borzicactus acanthurus (Vaupel) Britton \& Rose, $B$. eriotrichus (Werdermann \& Backeberg) Backeberg, $B$. faustianus Backeberg, B. keller-badensis Krainz, Cereus eriotrichus Werdermann \& Backeberg, Haageocereus convergens Ritter, $H$. eremiticus Ritter, $H$. faustianus (Backeberg) Ritter, $H$. imperialensis Ritter, $H$. neglectus Ritter, $H$. pacaranensis Ritter, $H$. pacaranensis var. tenuispinus Ritter, H. paradoxus Rauh \& Backeberg, $H$. peculiaris (Rauh \& Backeberg) Ritter, Loxanthocereus acanthurus (Vaupel) Backeberg, L. acanthurus var. ferox Backeberg, L. bicolor Ritter, L. brevispinus Rauh \& Backeberg, L. canetensis Rauh \& Backeberg, L. cantaensis Rauh \& Backeberg, L. convergens Ritter, L. cullmannianus Backeberg, L. eremiticus Ritter, L. eringes Rauh \& Backeberg, L. eriotrichus Backeberg, L. eulaliensis Rauh \& Backeberg, L. faustianus Backeberg, L. gracilispinus Rauh \& Backeberg, L. keller-badensis Backeberg \& Kraiz, L. multifloccosus Rauh \& Backeberg, L. neglectus Ritter, L. neglectus var. chimbotensis Ritter, L. pacaranensis Ritter, $L$. peculiaris Rauh \& Backeberg, L. pullatus Rauh \& Backeberg, L. pullatus var. brevispinus Rauh \& Backeberg, L. xylorhizus Ritter.

Planta arbustiva con ramas extendidas de $30 \mathrm{~cm}$ de longitud y 2 a $5 \mathrm{~cm}$ de diámetro que generalmente trepan, se arrastran o cuelgan de las rocas, a veces con pelos en el ápice; 15 a 18 costillas cortas y redondeadas, a veces divididas en afiladas muescas o pequeños tubérculos; 2 a 5 espinas centrales, amarillas al salir y marrones a grises al madurar de $1.5 \mathrm{~cm}$ de longitud; 20 espinas radiales, cortas, delgadas y amarillas al salir y marrones a grises al madurar, poco diferenciadas con respecto a las centrales; flor infundibuliforme de tubo recto o ligeramente curvado (zigomorfa), diurna, color escarlata, de 4 a $5 \mathrm{~cm}$ de longitud, polinizada por picaflores; pericarpelo desnudo o con pelos cortos; estambres numerosos exertos en un ciclo; gineceo de ovario ínfero con estilo exerto; fruto carnoso esférico o aovado de 2 a $2.5 \mathrm{~cm}$ de diámetro, pericarpo suave; semillas negras o mate. La especie se distribuye principalmente en los valles del departamento de Lima (Anderson, 2001; Ostolaza 1988). En la zona de estudio se determinó una subespecie.

Cleistocactus acanthurus subsp. faustianus (Backeberg) Ostolaza Cactaceae Consensus Initiatives 6:8. 1998. Borzicactus faustianus Backeberg. Kaktus ABC 193:411. 1936.

Sinónimo: Cleistocactus acanthurus var. faustianus (Backeberg) Ostolaza. Loxanthocereus faustianus (Backeberg) Backeberg.

Planta decumbente que alcanza los 40 a $45 \mathrm{~cm}$ de altura, ramificada desde la base, formando grupos de 3 a 5 tallos. Tallo terete de $30 \mathrm{~cm}$ de longitud y de 4 a 5 cm de diámetro, con 18 a 20 costillas, epidermis verde cubierta por areolas de forma redonda, de $0.1 \mathrm{~cm}$ de espesor y $0.3 \mathrm{~cm}$ de diámetro, color gris; espinas aciculares, 20 a 25 espinas por areola, de 1.5 a $2 \mathrm{~cm}$ de longitud, 0.05 a $0.1 \mathrm{~cm}$ de grosor, de color amarillento al aparecer y grisáceo al madurar; flores actinomorfas o zigomorfas, tubulares, rojo-anaranjadas, desarrollándose sobre las areolas floríferas, a lo largo del tallo, entre 6.5 y $8 \mathrm{~cm}$ de longitud y perianto de 2 a $5 \mathrm{~cm}$ de diámetro, con aproximadamente 34 tépalos: sepaloide, lanceolado-apiculado, de 1.8 a $2.5 \mathrm{~cm}$ de longitud y de 0.2 a $0.4 \mathrm{~cm}$ de ancho, y petaloides lingulados-rotundo, de 2.3 a $2.6 \mathrm{~cm}$ longitud y de 0.4 a $0.7 \mathrm{~cm}$ de ancho; tubo del receptáculo, de 3.5 a $4.5 \mathrm{~cm}$ de longitud y de 1 a $1.2 \mathrm{~cm}$ de diámetro, cubierto con brácteas a manera de escamas y pelos; cámara nectarial de 0.2 a $0.7 \mathrm{~cm}$ de longitud y de 0.2 a $0.3 \mathrm{~cm}$ de diámetro; androceo con aproximadamente 120 
estambres epipétalos exertos, entre 3.7 y 4.25 cm de longitud, anteras bitécicas entre $0.25 \mathrm{y}$ $0.35 \mathrm{~cm}$ de longitud y 0.1 cm de diámetro, amarillas, filamentos entre 1.4 y $3.9 \mathrm{~cm}$ de longitud y $0.025 \mathrm{~cm}$ de diámetro, color marfil; pericarpelo de 0.5 a 0.8 cm de longitud y de 0.6 a $0.7 \mathrm{~cm}$ de diámetro, cubierto con brácteas a manera de escamas y pelos; gineceo sincárpico de 5.4 a $7.8 \mathrm{~cm}$ de longitud, ovario ínfero de 0.3 a $0.6 \mathrm{~cm}$ de longitud y entre 0.3 a $0.4 \mathrm{~cm}$ de diámetro, 7 carpelos, numerosos óvulos de placentación parietal,

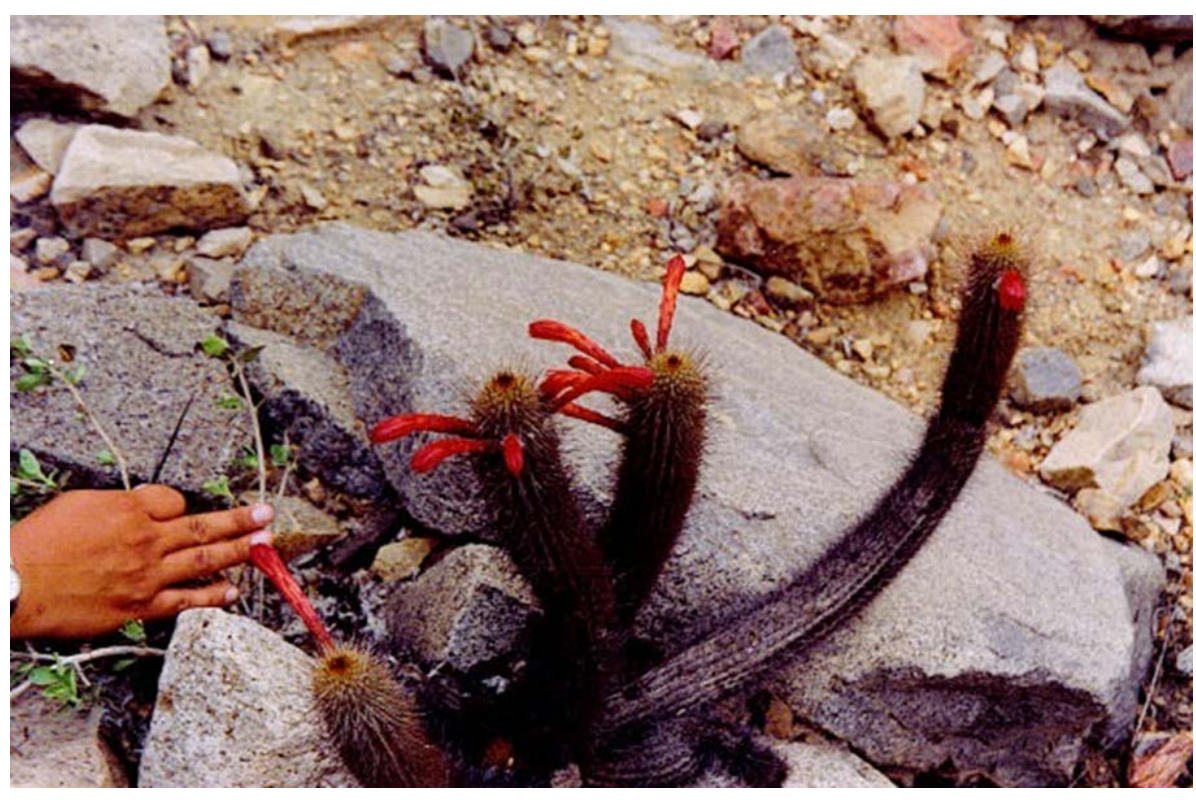

Figura 7. Cleistocactus acanthurus subsp. faustianus (Backeberg) Ostolaza. estilo de 4.6 a $6.5 \mathrm{~cm}$ de longitud y $0.05 \mathrm{~cm}$ de diámetro, estigma lobulado de 0.3 a $0.5 \mathrm{~cm}$ de longitud y de 0.2 a $0.6 \mathrm{~cm}$ de diámetro, 7 lóbulos color verde claro; fruto baya, de color verde, rojo, marrón o guinda, ovoide de 1.3 a $2.2 \mathrm{~cm}$ de longitud, 1.5 a $2 \mathrm{~cm}$ de diámetro mayor y $0.8 \mathrm{~cm}$ de diámetro menor, con flor persistente de 4.8 a $5 \mathrm{~cm}$ de longitud, funículo ariloso blanco cremoso desarrollado. Según Ramírez \& Ceroni (2009) las semillas son de forma reniformeovalada, con testa de color negro-marrón semimate, de tamaño mediano de $1.2 \mathrm{~mm}$ de longitud y $0.8 \mathrm{~mm}$ de ancho, con escultura de testa ruminada; la región hilomicropliar es grande y oblicua con el eje mayor de la semilla, con micrópilo e hilo grandes; los frutos presentan entre 400 a 1800 semillas (Figura 6). Esta subespecie se restringe a los valles de Chillón, Rímac y Santa Eulalia (Lima).

Material estudiado: LIMA: Cerro Umarcata, Santa Rosa de Quives, julio de 2003, 1260 msnm, Viviana Castro 112 (Herbario de Sitio Jardín Botánico OVN); Santa Rosa de Quives, abril de 1961, $\underline{P}$. Hutchinson 1033 (USM); Canta, julio de 2003, 1260 msnm, Mónica Arakaki, 1546a (USM); Huarochirí, octubre de 1980, Carlos Ostolaza, 048 (USM); Santa Rosa de Quives - Canta, febrero de 1953, R. Ferreyra, 8921 (USM) (Figura 7).

De acuerdo a Ostolaza (1988) la especie fue originalmente descrita por Vaupel en 1913 como Cereus acanthurus. Sin embargo, el género Cereus Miller 1754, se caracterizaba por tallos gruesos con costillas definidas y sus flores actinomorfas nocturnas generalmente blancas. Es por esto que Britton \& Rose (1919-1922) la incluyen dentro del género Borzicactus Riccobono 1909, que se caracteriza por sus tallos erectos o decumbentes y la flor anaranjada a escarlata,

de tubo estrecho y la nominan Borzicactus acanthurus (Vaupel) Britton \& Rose 1920. Sin embargo, también citan la existencia del género Cleistocactus Lemaire 1861, el cual se caracterizaba por sus tallos erectos o trepadores, flores muy finas, de estambres y estilo exertos y frutos con el perianto persistente. Ostolaza (1988) indica que en 1937 Backeberg publica el género Loxanthocereus que se caracteriza por sus flores zigomorfas color escarlata y allí incluye la especie en cuestión, nominándola Loxanthocereus acanthurus (Vaupel) Backeberg.

En una revisión de nomenclatura hecha por Hunt (1987, citado por Anderson (2001)) se rescata el género Cleistocactus debido a sus flores zigomorfas cuyos tubos parecen estar siempre cerrados y nomina la especie como Cleistocactus acanthurus (Vaupel) Hunt. Así mismo, establece que las diferencias morfológicas entre poblaciones son lo bastante evidentes como para reconocer la existencia de por lo menos tres subespecies. Una de estas fue tratada por Ostolaza (1996) como Cleistocactus acanthurus var. faustianus (Backeberg) Ostolaza, la cual se caracteriza por sus espinas más numerosas y más largas que las otras subespecies y por estar restringida a los valles del Rímac, Chillón y Santa Eulalia. Así, es luego tratada por Hunt (1999) y Hunt et al. (2006) como Cleistocactus acanthurus subsp. faustianus (Backeberg) Ostolaza y aunque Anderson (2001) la trata como Cleistocactus acanthurus (Vaupel) D.R. Hunt, indica la existencia de tres subespecies con diferentes características morfológicas y de hábitat, aunque remarca que es necesario mayor estudio del género y de sus variaciones en hábitat. Recientemente ha sido tratada por Ostolaza (2011) como Loxanthocereus acanthurus (Vaupel) Backeberg y 
subespecies que se distribuyen en los valles del Rímac, Chancay, Chillón y Cañete indicando que el uso del género Loxanthocereus Backeberg es debido a que tiene grandes flores diurnas rojas y zigomorfas, en contraste con las flores del género Cleistocactus Lemaire que son cerradas. La descripción de los individuos encontrados en la zona de estudio concuerda con las descripciones hechas para Cleistocactus acanthurus subsp. faustianus (Backeberg) Ostolaza por lo que se ha creído conveniente conservar esta nomenclatura cómo válida, la cuál ha sido corroborada con los nombre válidamente aceptados por las bases de datos TROPICOS (2014) e ITIS (2014).

Según Arakaki et al. (2006) esta especie es válida y presenta estado de conservación B1a-Amenazada No Evaluado; de acuerdo a la normatividad legal vigente, se encuentra en estado de Amenazada (EN) (D.S.043, 2006). Así mismo, ha sido tratada por Ostolaza \& Roque (2013) como una especie fragmentada en 3 subpoblaciones (que corresponden a las subespecies faustianus, pullatus y acanthurus) y ha sido considerada en estado de Casi Amenazada (NT), debido a que más del $20 \%$ del rango de distribución de la especie se ha perdido en los últimos 20 a 30 años debido al cambio de uso del suelo, aunque la población es estable. En Lomas, ha sido desplazada por el avance de la urbanización y las actividades agrícolas (Ostolaza \& Roque, 2013).

\section{Literatura citada.}

Anderson E.F. 2001. The Cactus Family. Timber Press, Inc. U.S.A.

Arakaki M., Ostolaza C., Cáceres F. \& Roque J. 2006. Cactaceae endémicas del Perú. En: El libro rojo de las plantas endémicas del Perú. León B., Roque J., UlloaUlloa C., Pitman N., Jørgensen P.M. \& Cano A. (eds.). Rev. Peru. Biol. Número especial 13(2): 193s - 219s. UNMSM.

Baker M.A, Mohlenbrock M.W. \& Pinkava D.J. 1985. A Comparison of Two New Methods of Preparing Cacti and Other Stem Succulents for Standard Herbarium Mounting. Taxon 34(1):118-120.

Barthlott W. \& Hunt R. 1993. Cactaceae. En: K. Kubitzki (Ed) The families and genera of vascular plants. Vol II: Flowering Plants. Dicotyledons. Springer-Verlag Berlin Heidelberg. Alemania. : 161-196.

Brako L. \& Zarucchi L. 1993. Catalogue of the Flowering Plants and Gymnosperms of Perú. Monogr. Syst. Bot. Missouri Botanical Garden 45:i-xl, 1-1286.

Britton N. \& Rose J. 1919-1922. The Cactaceae I, II, III \&IV. Carnegie Institution. U.S.A.

Cáceres F., Ostolaza C. \& Roque J. 2013. Espostoa melanostele. En: IUCN Red List of Threatened Species. V.2013.2. www.iucnredlist.org (29 Abril 2014).

Calderón Moya-Méndez N., Ceroni Stuva A. \& Ostolaza Nano C. 2004. Distribución y estado de conservación del género Haageocereus (Familia Cactaceae) en el departamento de Lima. Perú. Ecología Aplicada. 3 (1 y 2): 17 - 22.
Calderón N., Zappi D., Taylor N. \& Ceroni A. 2007.Taxonomy and conservation of Haageocereus Backeb.(Cactaceae) in Peru. Bradleya. 25: 45-124.

Castro V. 2006. Taxonomía de la familia Cactaceae en el valle de río Chillón, Lima: cerro Umarcata y quebrada Orobel. Tesis para optar el Grado Académico de Magister Scientiae. UNALM.

Ceroni A., Castro V., Calderón N. \& Novoa S. 2004. Cactario del Jardín Botánico "Octavio Velarde Núñez" de la Universidad Nacional Agraria La Molina. Quepo. Vol. 18: 34 - 38.

Ceroni Stuva A. \& Castro Cepero V. 2006. Los cactus del cerro Umarcata. Lima. Canta. Perú. Revista de Biología Augusto Weberbauer. 9: 15 - 18.

Decreto Supremo No43-2006-AG. Aprueban categorización de especies amenazadas de Flora Silvestre. Boletín de Normas Legales El Peruano. Norma aprobada el 6 de julio de 2006 y publicada el 13 de julio de 2006. Pp 323527-323528.

Hunt D. 1999. CITES Cactaceae Checklist. Royal Botanic Gardens Kew \& International Organization for Succulent Plant Study. England.

Hunt D., Taylor N. \& Charles G. 2006. The New Cactus Lexicon. DH Books. U.K.

Integrated Taxonomic Information System (ITIS). 2014. System on-line Database. www.itis.gov (29 Abril 2014).

Novoa S., Ceroni A. \& Arellano C. 2005a. Contribución al conocimiento de la fenología del cactus Neoraimondia arequipensis subsp. roseiflora (Werderm. \& Backeb.) Ostolaza (Cactaceae) en el valle del río Chillón, LimaPerú. Ecología Aplicada. 4 (1 y 2): 35-40.

Novoa S., Redolfi I. \& Ceroni A. 2005b. Patrón de actividad diario de la hormiga Camponotus sp. en los botones florales del cactus Neoraimondia arequipensis subsp. roseiflora (Werderm. \& Backeb.) Ostolaza. Ecología Aplicada. 4 (1 y 2): 77-81.

Novoa S., Redolfi I., Ceroni A. \& Arellano C. 2005c. El forrajeo de la hormiga Camponotus sp. en los botones florales del cactus Neoraimondia arequipensis subsp. roseiflora (Werderm. \& Backeb.) Ostolaza (Cactaceae). Ecología Aplicada. 4 (1 y 2): 83-90.

Missouri Botanical Garden. 2014. Tropicos. www.tropicos.org (29 abril 2014).

Ostolaza C. 1988. Loxanthocereus acanthurus (Vpl.) Backbg. Cactus and Succulent Journal. 60(1):205-208. U.S.A.

Ostolaza C. 1996. A closer look at the conservation status of Cacti in the vicinity of Lima, Peru. British Cactus and Succulent Journal. 14(4): 158-174.

Ostolaza C. 2011. 101 Cactus del Perú. MINAM. 253pp.

Ostolaza C. \& Cáceres F. 2013. Mila caespitosa. En: IUCN 2013 Red List of Threatened Species. V.2013.2. www.iucnredlist.org (29 Abril 2014).

Ostolaza C., Ceroni A., Calderón N. \& Novoa S. 2005. The Huaura Valley, Lima, Peru - revisited. British Cactus and Succulent Journal. 23(1): 25-33.

Ostolaza C., Ceroni A., Dreyfus G., Calderón N. \& Novoa S. 2003. Cacti of the Huaura river valley, Peru - a conservation study. British Cactus and Succulent Journal. 21(2):87-97. U.K.

Ostolaza C. \& Roque J. 2013. Cleistocactus acanthurus. En: IUCN 2013 Red List of Threatened Species. V.2013.2. www.iucnredlist.org (29 Abril 2014). 
Ramírez N. \& Ceroni A. 2009. Caracterización morfológica de semillas de cactáceas del Cerro Umarcata, Canta, Lima, Perú. Quepo. 23: 90-98.

Taylor N.P. \& Zappi D.C. 2004. The Cacti of eastern Brazil. Royal Botanic Gardens of Kew. Richmond, U.K.

Teixeira V., Castro V., Ceroni A. \& Eyzaguirre R. 2004 Diversidad y densidad de la comunidad de cactáceas en el valle del río Chillón: cerro Umarcata y quebrada
Orobel y su relación con los factores edáficos. Ecología Aplicada. 3 (1 y 2): 1-8.

Wallace R.S. \& Gibson A.C. 2002. Evolution and Systematics: 1-21. In: Nobel, P.S. Cacti: Biology and Uses. Univ. California Press. Berkeley.

Weberbauer A. 1945. El mundo vegetal de los Andes Peruanos: Estudio fitogeográfico. 2da Edición. Estación Experimental Agrícola de La Molina. Dirección de Agricultura. Ministerio de Agricultura. Lima.

\section{Anexo: Figuras pares.}
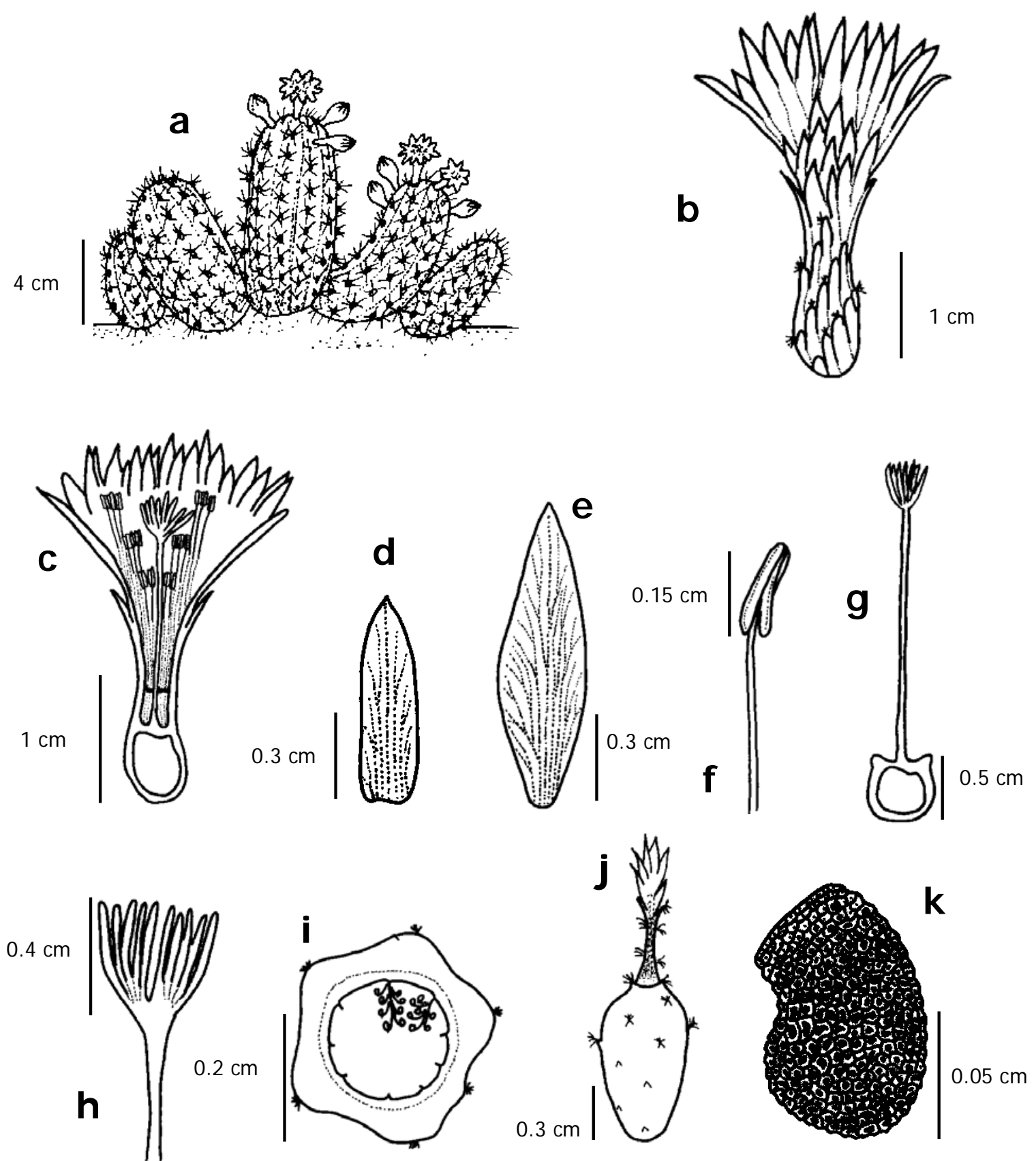

Figura 2. Mila nealeana Backeberg. a. Hábito; b. Flor; c. Sección longitudinal de la flor; d. Tépalo sepaloide; e. Tépalo petaloide; f. Estambre; g. Gineceo; h. Estigma; i. Sección transversal del ovario; j. Fruto; k. Semilla. 

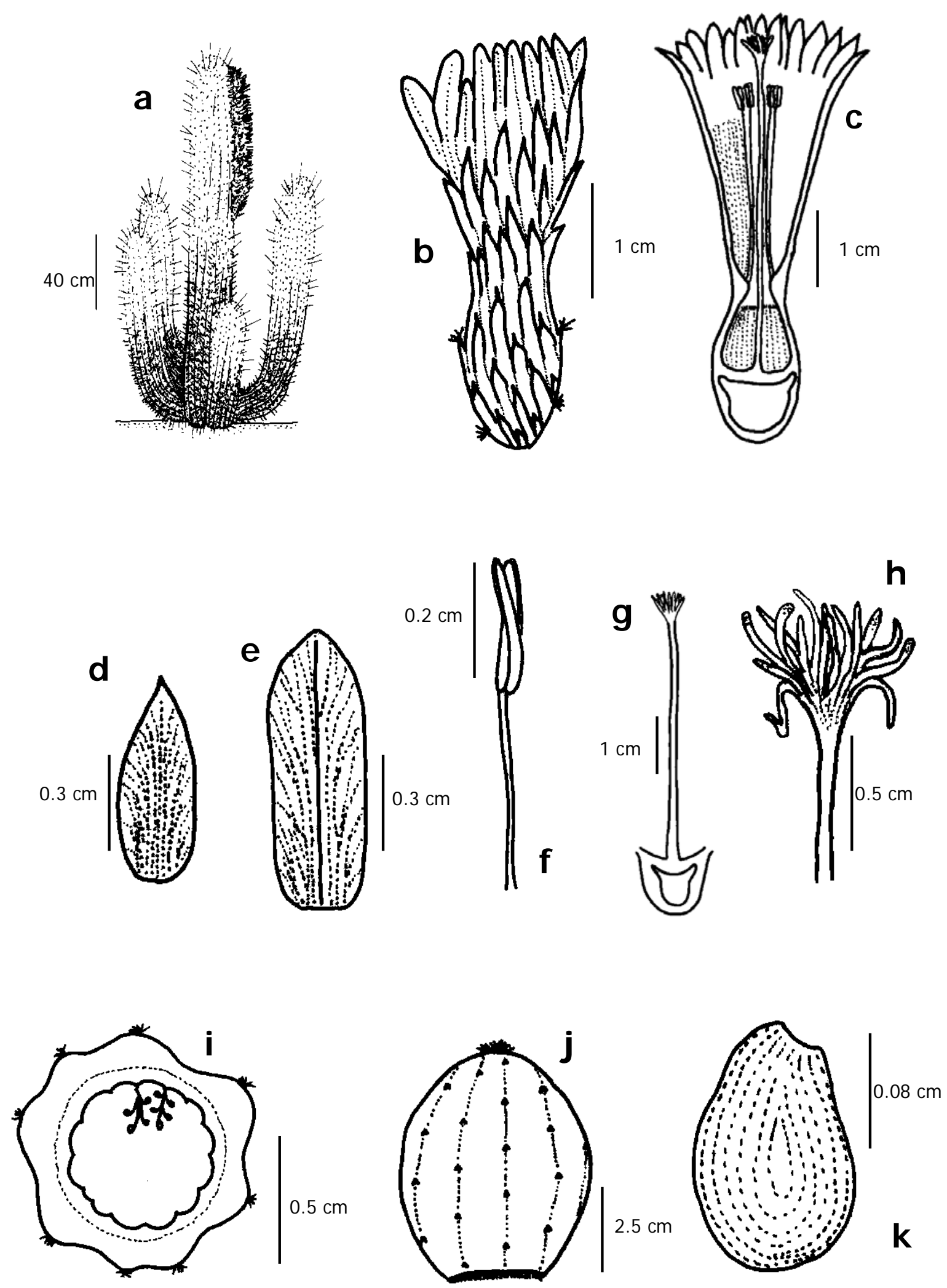

Figura 4. Espostoa melanostele (Vaupel) Borg. a. Hábito; b. Flor; c. Sección longitudinal de la flor; d. Tépalo sepaloide; e. Tépalo petaloide; f. Estambre; g. Gineceo; h. Estigma; i. Sección transversal del ovario: i. Fruto: k. Semilla . 

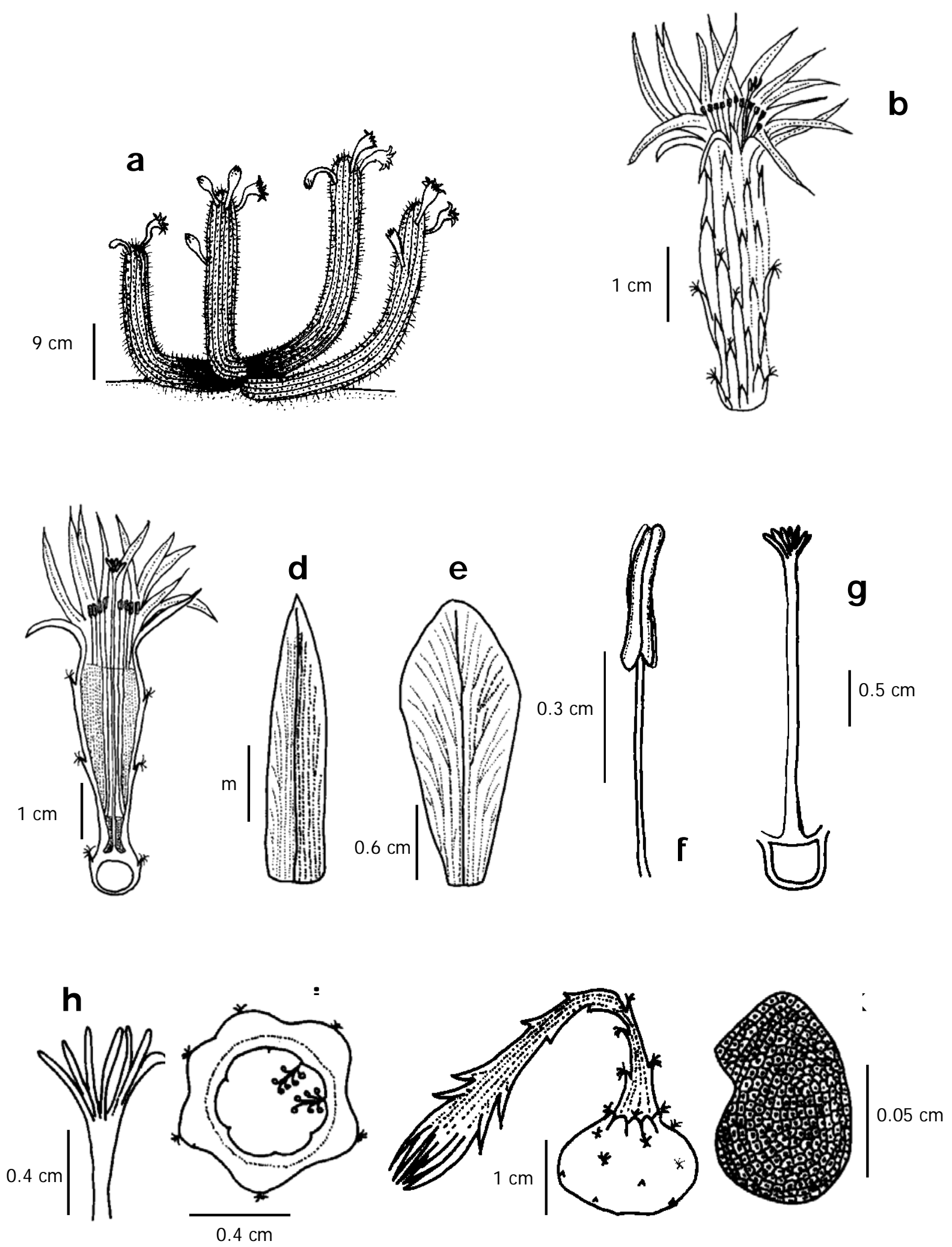

Figura 6. Cleistocactus acanthurus subsp. faustianus (Backeberg) Ostolaza. a. Hábito; b. Flor; c. Sección longitudinal de la flor; d. Tépalo sepaloide; e. Tépalo petaloide; f. Estambre; g. Gineceo; h. Estigma; i. Sección transversal del ovario: i. Fruto: k. Semilla. 
Enero - Julio 2015

1 Jardín Botánico “Octavio Velarde Núñez”. Departamento Académico de Biología, Universidad Nacional Agraria La Molina. Av. La Molina s/n, Lima 12, Perú. Apartado postal: 12-056, Perú. Correo electrónico: vcastro@lamolina.edu.pe.

2 Jardín Botánico “Octavio Velarde Núñez”. Departamento Académico de Biología, Universidad Nacional Agraria La Molina. Av. La Molina s/n, Lima 12, Perú. Apartado postal: 12-056, Perú. Correo electrónico: aceroni@lamolina.edu.pe. 